\title{
Functional magnetic resonance imaging response as an early biomarker of cognitive decline in elderly patients with metabolic syndrome
}

\author{
Nadia Shigaeff ${ }^{\mathrm{a}, *}$, Edson Amaro ${ }^{\mathrm{b}}$, Fabio G.M. Franco ${ }^{\mathrm{c}}$, Alessandro F. Jacinto ${ }^{\mathrm{d}}$, \\ Gabriela Chiochetta $^{e}$, Maysa S. Cendoroglo ${ }^{f}$, Vanessa A. Citero ${ }^{\mathrm{e}}$ \\ a Psychiatry Department, Escola Paulista de Medicina, Universidade Federal de São Paulo and Hospital Israelita Albert Einstein, Rua Borges Lagoa, 570, CEP:04038-030, \\ São Paulo, SP, Brazil \\ b Hospital Israelita Albert Einstein, Avenida Albert Einstein, 627, Sao Paulo, Brazil \\ ${ }^{\mathrm{c}}$ Hospital Israelita Albert Einstein, Rua Madre Cabrini, 462, CEP:04020-001, São Paulo, SP, Brazil \\ d Psychiatry Department, Escola Paulista de Medicina, Universidade Federal de São, Paulo and Internal Medicine Department, Faculdade de Medicina de Botucatu, \\ Universidade Estadual de Sao Paulo Julio de Mesquita Filho, Rua Borges Lagoa, 570, CEP:04038-030, São Paulo, SP, Brazil \\ e Psychiatry Department, Escola Paulista de Medicina, Universidade Federal de São Paulo, Rua Borges Lagoa, 570, CEP:04038-030, São Paulo, SP, Brazil \\ ${ }^{\mathrm{f}}$ Geriatric Division - Internal Medicine Department, Escola Paulista de Medicina, Universidade Federal de São Paulo, Rua Francisco de Castro, 105, CEP:04020-050, São \\ Paulo, SP, Brazil
}

\section{A R T I C L E I N F O}

\section{Keywords:}

Metabolic syndrome

Elderly

Biomarker

Neuropsychological assessment

Cognitive impairment

Functional magnetic resonance

\begin{abstract}
A B S T R A C T
Objectives: We assessed whether potential changes in brain activation patterns of elderly individuals with metabolic syndrome (MetS) who were cognitively healthy (without mild cognitive impairment or dementia) were associated with cognitive decline in executive function in the short-term.

Method: We analyzed 43 individuals (23 MetS, 20 controls) using a global geriatric evaluation, a neuropsychological battery, and task-related (attention) fMRI exam. Correlation analysis between the fMRI signal at baseline and cognitive impairment after 1 year was based on the voxel-based Pearson coefficient, corrected for multiple comparisons.

Results: At baseline, MetS patients showed reduced brain response in frontal and parietal regions compared to controls. After one year, the MetS group also showed a decline in verbal fluency performance. fMRI response in the right dorsolateral prefrontal cortex and bilateral parietal lobes was negatively correlated with verbal fluency decline in the MetS group.

Discussion: Our results provide an early biomarker of the possible development of cognitive impairment, particularly in the executive function, of elderly individuals suffering from MetS. These findings also point to an up or down regulation which could be interpreted as compensatory mechanism for possible brain tissue burden caused by MetS.
\end{abstract}

\section{Introduction}

Metabolic Syndrome (MetS) involves a series of clinical and laboratory abnormalities that increase the risk of cardiovascular disease. MetS has been best described as a set of symptoms that, altogether, are associated with greater cardiovascular risk compared to the risk of each factor in isolation (Rigo, Vieira, Dalacorte, \& Reichert, 2009).

Although much research has evaluated the association of MetS with cardiovascular risk, particularly cerebrovascular accidents (CVA) (De Silva et al., 2009; Haley, Gonzales, Tarumi, \& Tanaka, 2012; Park \& Kwon, 2008; Zhang et al., 2010), there is growing interest in the potential impact of MetS on cognitive impairment. In fact, elderly individuals diagnosed with MetS have shown poorer scores on cognitive tests compared to matched adults without MetS (Collinson, Tong, Loh, Chionh, \& Merchant, 2014; Rouch et al., 2014), especially MetS patients with higher inflammatory activity (Cavalieri et al., 2010; Dik et al., 2007; Roberts et al., 2010). Similar findings were obtained with longitudinal studies using screening tests, which have shown a gradual development from normal to mild cognitive impairment (MCI) or even direct evolution of MCI to dementia in elderly individuals with MetS followed from two to 16 years (Bruce et al., 2008; Creavin et al., 2012; Luchsinger, Tang, Stern, Shea, \& Mayeux, 2001; McEvoy et al., 2012; McGuire, Ford, \& Ajani, 2006; Niwa et al., 2006; Raffaitin et al., 2011)

However, the extent to which the risk of cognitive impairment in

\footnotetext{
* Corresponding author.

E-mail addresses: nadia.shigaeff@yahoo.com.br, nadia.shigaeff@einstein.br (N. Shigaeff), edsonjr@einstein.br (E. Amaro), ffranco@einstein.br (F.G.M. Franco),

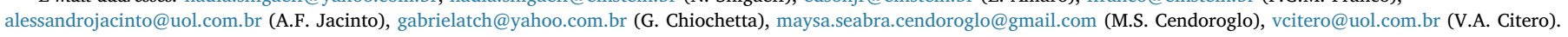


Table 1

Cognitive Performance of elderly individuals with and without MetS.

\begin{tabular}{|c|c|c|c|c|c|c|c|c|}
\hline & \multirow[t]{3}{*}{ Moment } & \multicolumn{6}{|l|}{ Group } & \multirow[t]{3}{*}{$\mathrm{P}$} \\
\hline & & \multicolumn{3}{|l|}{ Control } & \multicolumn{3}{|l|}{ MetS } & \\
\hline & & Median & $1^{\circ}$ quartile & $3^{\circ}$ quartile & Median & $1^{\circ}$ quartile & $3^{\circ}$ quartile & \\
\hline \multirow[t]{2}{*}{ Sustained Attention (z-escore) } & Baseline & $-2,2$ & $-4,9$ & $-0,3$ & $-3,7$ & $-6,3$ & $-1,4$ & 0,099 \\
\hline & One year follow-up & $-2,5$ & $-5,1$ & $-0,8$ & $-3,0$ & $-5,4$ & $-1,0$ & 0,448 \\
\hline \multirow[t]{2}{*}{ Switch Attention (z-escore) } & Baseline & $-2,9$ & $-5,9$ & $-1,2$ & $-4,2$ & $-7,4$ & $-2,1$ & 0,244 \\
\hline & One year follow-up & $-3,2$ & $-6,7$ & $-1,4$ & $-4,0$ & $-6,9$ & $-2,4$ & 0,385 \\
\hline \multirow[t]{2}{*}{ Work Memory (z-escore) } & Baseline & 0,7 & $-0,6$ & 1,5 & 0,7 & 0,0 & 2,3 & 0,365 \\
\hline & One year follow-up & 0,0 & $-0,9$ & 1,5 & 0,7 & $-0,9$ & 1,3 & 0,821 \\
\hline \multirow[t]{2}{*}{ Immediate Memory (z-escore) } & Baseline & 1,3 & $-0,4$ & 2,2 & 1,3 & 0,4 & 2,2 & 0,976 \\
\hline & One year follow-up & 1,3 & 0,4 & 2,2 & 1,3 & 0,4 & 2,2 & 0,794 \\
\hline \multirow[t]{2}{*}{ Mental Flexibility (z-escore) } & Baseline & $-0,5$ & $-0,5$ & 0,5 & $-1,0$ & $-1,0$ & 1,0 & 0,172 \\
\hline & One year follow-up & $-1,2$ & $-1,8$ & $-0,6$ & $-1,8$ & $-1,8$ & 0,0 & 0,753 \\
\hline \multirow[t]{2}{*}{ Long memory - immediate recall (z-escore) } & Baseline & $-0,1$ & $-1,5$ & 0,5 & 0,0 & $-1,5$ & 0,3 & 0,899 \\
\hline & One year follow-up & 0,2 & $-1,1$ & 1,3 & $-0,3$ & $-1,3$ & 0,5 & 0,198 \\
\hline \multirow[t]{2}{*}{ Long memory - delayed recall (z-escore) } & Baseline & $-0,1$ & $-0,9$ & 0,6 & $-0,6$ & $-1,2$ & 0,3 & 0,250 \\
\hline & One year follow-up & 0,2 & $-0,9$ & 0,6 & $-0,1$ & $-1,0$ & 1,1 & 0,982 \\
\hline \multirow[t]{2}{*}{ Long memory - recognition (z-escore) } & Baseline & $-0,4$ & $-1,2$ & 0,2 & $-0,8$ & $-2,8$ & 0,2 & 0,198 \\
\hline & One year follow-up & $-0,6$ & $-1,4$ & 0,3 & $-0,4$ & $-2,6$ & 0,7 & 0,691 \\
\hline \multirow[t]{2}{*}{ Executive Function (z-escore) } & Baseline & $-0,7$ & $-2,3$ & $-0,3$ & $-1,3$ & $-3,6$ & $-0,2$ & 0,546 \\
\hline & One year follow-up & $-0,6$ & $-2,5$ & $-0,1$ & $-2,8$ & $-4,6$ & $-0,3$ & 0,128 \\
\hline \multirow[t]{2}{*}{ Constructive praxis (weighted) } & Baseline & 10,0 & 8,0 & 12,0 & 9,0 & 7,0 & 12,0 & 0,680 \\
\hline & One year follow-up & 9,5 & 8,0 & 11,2 & 8,0 & 8,0 & 11,7 & 0,348 \\
\hline \multirow[t]{2}{*}{ Naming ability (z-escore) } & Baseline & 0,0 & $-0,2$ & 0,5 & 0,1 & $-1,2$ & 0,4 & 0,213 \\
\hline & One year follow-up & 0,4 & $-0,1$ & 0,7 & 0,3 & $-0,5$ & 0,7 & 0,384 \\
\hline \multirow[t]{2}{*}{ Verbal fluency (z-escore) } & Baseline & $-0,1$ & $-0,8$ & 0,4 & $-0,4$ & $-0,9$ & 0,0 & 0,100 \\
\hline & One year follow-up & 0,0 & $-0,5$ & 0,6 & $-0,4$ & $-0,9$ & $-0,2$ & 0,017 \\
\hline
\end{tabular}

individuals with MetS is associated with functional changes in brain activity is not well established, and the impact of these changes on cognitive performance in the long-term is still unclear. To our knowledge, there have been only three studies that have explored this issue to date. A cross-sectional study concluded that MetS in elderly individuals without dementia or a history of stroke was associated with cognitive impairment, especially impairment of memory and executive function. Individuals with higher inflammatory activity also had higher chances of developing cognitive impairment; however, interestingly, there was no structural or functional abnormality in magnetic resonance imaging (MRI) (Cavalieri et al., 2010), even though ischemic alterations in cerebral tissue resulting from stroke are associated with cognitive impairment.

The second study, and the only one involving functional neuroimaging in MetS in a population without a history of vascular events, evaluated a sample of elderly patients with MetS and controls by BOLD (blood oxygen level dependent) effect during a working memory task. Patients with MetS showed a lower BOLD signal in the right superior frontal gyrus and in the right superior and left inferior parietal lobes during the task (Hoth et al., 2011), indicating a failure to produce the expected hemodynamic response in these regions. Lastly, we have previously shown in a cross-sectional study that MetS patients had a discrete reduction in performance in a specific attention task and reduced response in BOLD signal in the parietal and occipital lobes compared with matched controls (Shigaeff et al., 2012). However, the patients were considered cognitively healthy because they did not meet the criteria for mild cognitive impairment (MCI) or dementia. These results point to possible brain changes caused by MetS, and the possibility of progression to preventable neurovascular and cognitive damage in the long-term.

Because these studies were either restricted to a population with a history of stroke, examined only changes in memory function or employed cross-sectional design with no follow up of patients, it is not yet clear whether functional abnormalities in brain activation patterns can be used as an early marker of impairment risk across cognitive domains in elderly individuals with MetS. This is particularly important in light of the higher risk of neurodegenerative disease in patients with MetS.

In this study we aimed to evaluate whether brain activation patterns in cognitively healthy (without MCI or dementia) elderly individuals with MetS differ from those of matched healthy controls. We also evaluated whether these potential differences in activation pattern could predict cognitive decline in the short term. By showing that the MetS group exhibited reduced brain activation at baseline while having the same neuropsychological performance as controls, our results should help in the early diagnosis of neurodegenerative pathologies in this population. Indeed, we show that reduced pre-frontal fMRI signal at baseline is negatively associated with reduced verbal fluency performance after 1 year.

\section{Methods}

This was a cohort study with one-year follow-up of Brazilian elderly subjects from two sites: volunteers from the public health system attending the geriatrics and gerontology outpatient clinic of the Universidade Federal de Sao Paulo, and healthy elderly individuals from the Serviço Social do Comércio (Commerce Social Service Units), both in Sao Paulo. The assessments occurred between April 2010 and March 2013.

The inclusion criteria were: (1) age $\geq 65$ years; (2) schooling level between one and four years; and (3) score on the Mini-Mental State Examination $\geq 22$, as suggested by Brazilian validation (Brucki, Nitrini, Caramelli, Bertolucci, \& Okamoto, 2003). Using this criteria, in both groups there are no subject with MCI. Exclusion criteria were: (1) 

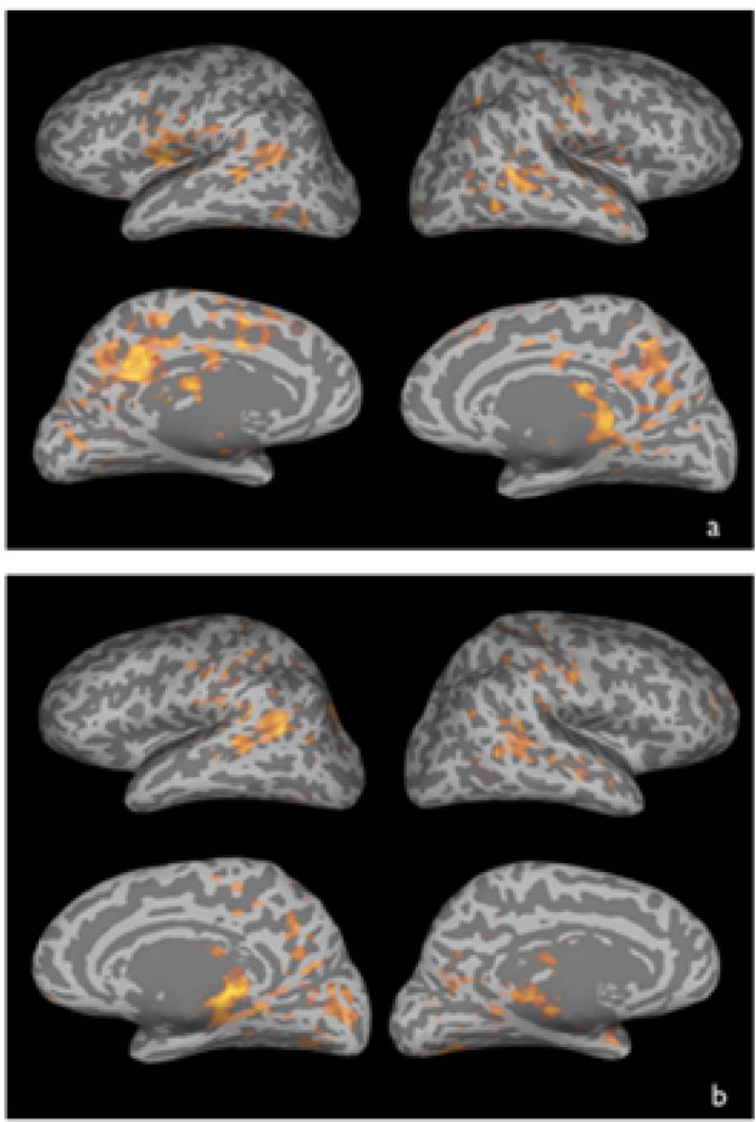

Fig. 1. Statistical brain activation map. Areas showing reduced activity in the MetS group $(\mathrm{n}=23)$ in the [incongruent $>$ neutral] contrast (a) and the [incongruent $>$ congruent] contrast (b). Clusters were found in the bilateral dorsolateral prefrontal cortex (inferior and middle frontal gyrus), bilateral intraparietal sulcus, and anterior cingulated gyrus in contrasts (a) and (b); see Table 3 for more detail. ANOVA, p $<0.05$, FDR corrected.

Table 2

Brain areas with reduced BOLD response in the MetS group compared with the control group.

\begin{tabular}{|c|c|c|c|c|c|}
\hline \multirow[t]{2}{*}{ Description } & \multirow[t]{2}{*}{ Side } & \multirow[t]{2}{*}{ Z-score } & \multicolumn{3}{|c|}{ Talairach Cood (X Y Z) } \\
\hline & & & $\mathrm{X}$ & $\mathrm{Y}$ & $\mathrm{Z}$ \\
\hline \multicolumn{6}{|l|}{ Incongruent > Neutral } \\
\hline Middle Occipital Gyrus & $\mathrm{R}$ & 5.34 & 48.2 & -69.3 & 5.0 \\
\hline Medial Frontal Gyrus & $\mathrm{L}$ & 4.00 & -6.0 & 35.1 & -13.0 \\
\hline Temporo-Occipital & $\mathrm{L}$ & 4.39 & -46.2 & -55.2 & -5.0 \\
\hline Postcentral Gyrus & $\mathrm{L}$ & 4.32 & -60.2 & -29.1 & 17.0 \\
\hline Lentiform Nucleus & $\mathrm{L}$ & 4.17 & -18.1 & -3.0 & -3.0 \\
\hline Transverse Temporal Gyrus & $\mathrm{R}$ & 4.11 & 54.2 & -15.1 & 9.0 \\
\hline Inferior Parietal Lobule & $\mathrm{R}$ & 4.04 & 54.2 & -33.1 & 47.0 \\
\hline Cingulate Gyrus & $\mathrm{R}$ & 3.79 & 18.1 & -37.1 & 43.0 \\
\hline Posterior Cingulate & $\mathrm{R}$ & 4.76 & 6.0 & -49.2 & 15.0 \\
\hline Superior Parietal Lobule & $\mathrm{R}$ & 3.94 & 10.0 & -77.3 & 43.0 \\
\hline \multicolumn{6}{|l|}{ Incongruent $>$ Congruent } \\
\hline Precuneus & $\mathrm{L}$ & 5.31 & -12.0 & -77.3 & 49.0 \\
\hline Cerebellum & $\mathrm{L}$ & 4.88 & -16.1 & -83.3 & -39.0 \\
\hline Middle Temporal Gyrus & $\mathrm{R}$ & 4.68 & 48.2 & -69.3 & 7.0 \\
\hline Middle Occipital Gyrus & $\mathrm{L}$ & 4.63 & -40.2 & -73.3 & 5.0 \\
\hline Superior Parietal Lobule & $\mathrm{R}$ & 4.52 & 20.1 & -65.3 & 29.0 \\
\hline Medial Frontal Gyrus & & 4.31 & -2.0 & -15.1 & 57.0 \\
\hline Supramarginal Gyrus & $\mathrm{L}$ & 4.27 & -62.2 & -43.2 & 29.0 \\
\hline Postcentral Gyrus & $\mathrm{R}$ & 4.11 & 50.2 & -31.1 & 39.0 \\
\hline Superior Frontal Gyrus (rDLPFC) & $\mathrm{R}$ & 4.02 & 28.1 & 43.2 & 37.0 \\
\hline Transverse Temporal Gyrus & $\mathrm{R}$ & 3.74 & 46.2 & -35.1 & 1.0 \\
\hline
\end{tabular}

$\mathrm{p}<0.05$, corrected; R: right; L: left; rDLPFC: right dorsolateral prefrontal cortex; Talairach coordinates indicate local maximum response in MNI-305 template. FDR methods to correct for false positive. having a clinical history of cerebral disease; (2) clinically suspected of having cognitive impairment; and (3) having a psychiatric history that could explain cognitive impairment due to other causes (history of alcohol abuse, schizophrenia, or mental deficit). Neuroimaging data was analyzed by a board-certified radiologist in order to exclude participants with incidental findings in the structural MR data. The diagnosis of MetS was reached according to the criteria of the National Cholesterol Education Program, Adult Treatment Panel III (NCEP ATPIII) (Ford, Giles, \& Dietz, 2002; Grundy et al., 2005). The study was approved by the Research Ethics Committee of the Universidade Federal de São Paulo (CEP - 0306/10) and the Hospital Israelita Albert Einstein (CEP - 09/1154). All participants signed a free and informed consent form.

\subsection{Participants}

A total of 62 elderly individuals were recruited by physician from a geriatrics outpatient clinic of the Universidade Federal de São Paulo. The patients in the control group were selected through face-to-face speeches about aging offered to the community by our researcher team. After the speeches the elderly were invited to participate in the study. Fifty volunteers were included in the study and 12 were excluded according to the criteria outlined above. The baseline study consisted of clinical evaluation and neuropsychological assessment of 25 cases diagnosed with MetS and 25 controls accomplished by neuropsychologist. The fMRI data from three subjects ( 2 MetS, 1 control) was dismissed during image processing (due to technical problems and/or head movement artifacts), and one subject from the control group did not agree to participate in the fMRI experiment. Thus, the fMRI analysis comprised 23 MetS subjects and 23 controls.

At the follow-up time, all participants were invited to continue their participation by telephone call. Then, after one year, only 43 older adults (23 MetS and 20 controls) remained in this study due to one death and two drop-outs. There were no differences between the final sample and the drop outs in any parameter measured.

The two groups were paired for those variables, as age group over 60 years, educational level and history of systemic arterial hypertension (SAH). The elderly with hypertension were equally distributed among the groups, due to the high prevalence of this morbidity in the elderly population.

\subsection{Neuropsychological assessment}

The researcher who made the neuropsychological assessment was blind to the group selection of the volunteers. This assessment included: immediate memory, working memory, long-term memory (episodic and semantic), language, praxis, attention, mental flexibility, and executive function.

Neuropsychological evaluations were performed during single exams that lasted about two hours. The tests were randomized during each exam to avoid bias. For more detailed information about that neuropsychological assessment, see Shigaeff et al. (2013).

\subsection{Stroop word-color task}

Before starting the fMRI exam, volunteers were familiarized with the console and the tasks that would be performed during image acquisition. The console had three buttons (blue, red and green), and was positioned at the right hand of the participant. The fingers were arranged with one on each button (index, middle, and ring).

The task was an adaptation of the original Stroop test (Golden \& Freshwater, 2002). In this version, subjects were instructed to look at and focus their attention on the center of a screen and, when prompted, to press the button whose color matched that of a word shown on the screen. Initially, and between each word, a black screen was presented with a white cross in the center. Words were presented in 
Verbal Fluency Change in $1 y$ and Brain Activation: all areas

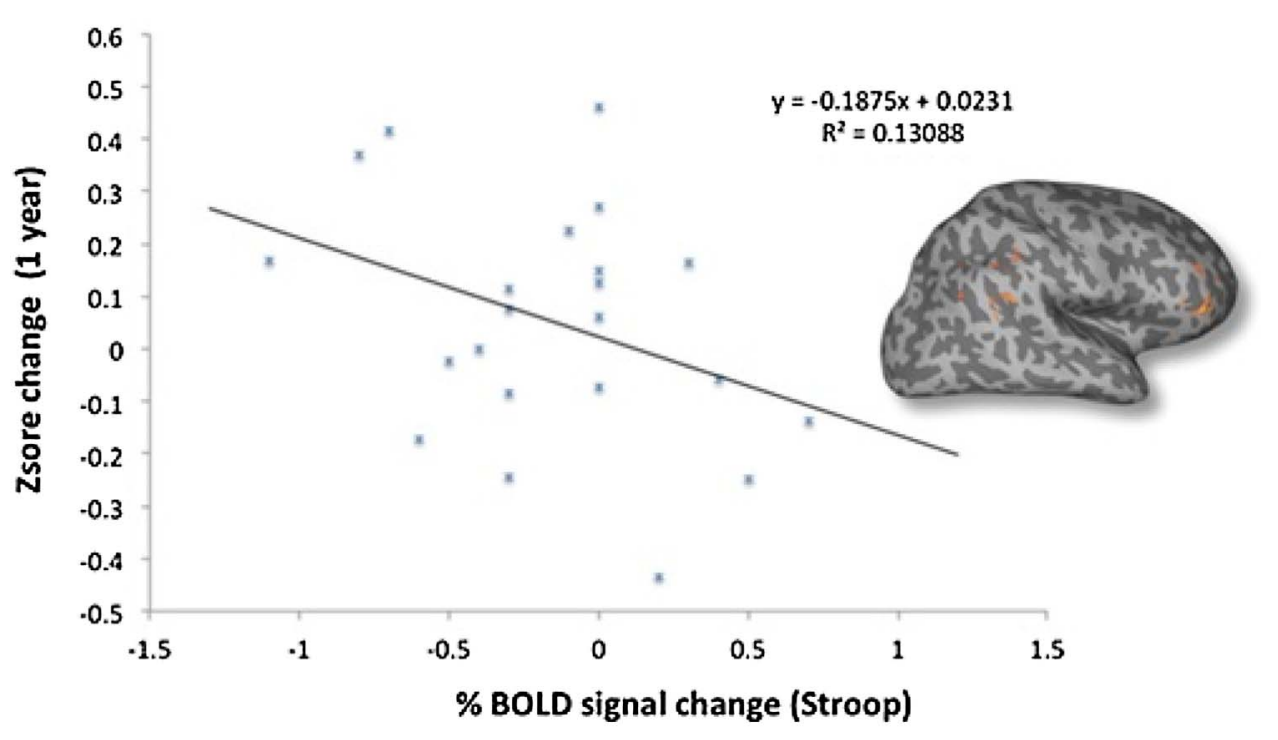

Fig. 2. Changes in verbal fluency and brain activation. Scatterplot showing fMRI data (BOLD\% signal change from incongruent trials vs. congruent trials) and verbal fluency (FAS test) z-score changes in one year (Pearson correlation coefficient, $\mathrm{p}<0.005$, $\mathrm{R}^{2}=0.13$, corrected).

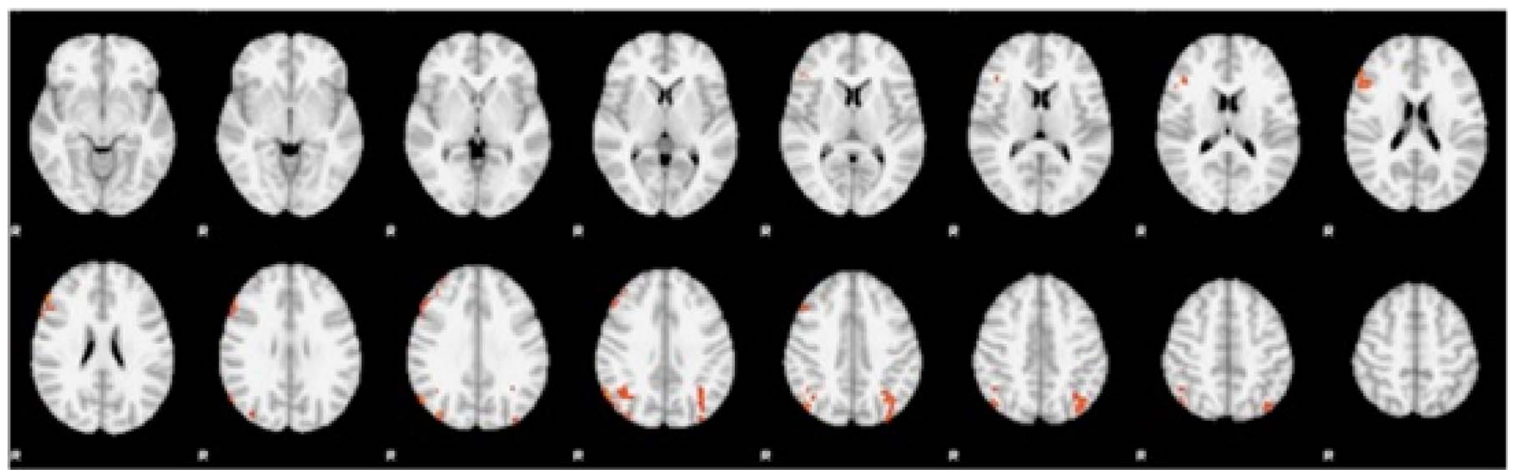

Fig. 3. Areas with brain activity correlated with cognitive decline after 1 year.

Table 3

Brain areas showing correlation between the BOLD response and cognitive decline (verbal fluency) in the MetS group after 1 year.

\begin{tabular}{llllll}
\hline Description & Side & Z-score & \multicolumn{3}{l}{ Talairach Cood (X Y Z) } \\
\cline { 3 - 6 } & & & $\mathrm{X}$ & $\mathrm{Y}$ & $\mathrm{Z}$ \\
\hline Middle Frontal Gyrus (rDLPFC) & $\mathrm{R}$ & 5.09 & 52.2 & 33.1 & 23.0 \\
Parietal Lobule & $\mathrm{R}$ & 3.75 & 56.2 & -59.2 & 37.0 \\
Parietal Lobule & $\mathrm{L}$ & 3.59 & -38.1 & -73.3 & 47.0 \\
\hline
\end{tabular}

Pearson correlation coefficient, $\mathrm{p}<0.05$, corrected; R: right; L: left; rDLPFC: right dorsolateral prefrontal cortex; Talairach Coordinates indicate local maximum response in MNI-305 template.

sequence with three conditions: (1) congruent stimuli, in which a color name was presented in its own color, e.g., the word 'blue' in blue letters (in which case the subject must press the blue button); (2) neutral stimuli, in which any word (except for a color name) was presented in one of three colors (blue, red and green), e.g., the word 'house' in red letters (in which case the subject must press the red button); and (3) incongruent stimuli, in which a color name was presented in a color different from the one named, e.g, the word 'red' in blue letters (in which case the subject must press the blue button).

We collected two fMRI runs, each comprising five blocks of each condition (10 trials per block, with each trial consisting of a 2-s word presentation followed by a 1-s fixation cross). Participants rested in the MR scanner during the time between the two runs while the structural MR data was acquired.

\subsection{Image acquisition}

All fMRI exams were performed on the same equipment from Siemens (Erlenger, Germany), with a $3.0 \mathrm{~T}$ main field, gradients of $45 \mathrm{mT} / \mathrm{m}$, and using the same version (B16) of the MR system software package.

The exclusion criteria for the neuroimaging examination were: the candidate was unable to perform magnetic resonance imaging, due to contraindication for examination, such as claustrophobic behavior, brain clips, among others.

The procedure for fMRI acquisition was previously described by our group. Briefly, fMRI acquisition was based on T2*-weighted echo planar (EPI) images for whole brain (EPI GRE T2-BOLD PACE: TR $=2000 \mathrm{~ms}$, $\mathrm{TE}=30 \mathrm{~ms}$, 32 slices, with 3.3-mm isotropic voxels). A total of $180 \mathrm{vol}$ were used to obtain the MRI signal during the fMRI Stroop task acquisition, lasting $5 \mathrm{~min} 4 \mathrm{~s}$ (Kozasa et al., 2012). We also collected a MPRAGE T1-weighted 3D sequence (1-mm isotropic voxels, $\mathrm{TR}=2500 \mathrm{~ms}, \quad \mathrm{TE}=3.45 \mathrm{~ms}, \quad \mathrm{FOV}=265 \mathrm{~mm}, \quad$ inversion time $=1100$, flip angle 7 degrees) for image registration to the MNI template (see below).

\subsection{Image processing}

The processing of the fMRI data was carried out using FSL version 4.1 (FMRIB, Analysis Group, Oxford, UK, http://www.fmrib.ox.ac.uk/ $\mathrm{fsl} /$ ). Initially, the main EPI images were pre-processed for movement correction (MCFLIRT), spatial smoothing with Gaussian function $($ FWHM $=5 \mathrm{~mm})$, and spatial normalization to standard space 
(MNI305). Activation maps were produced using the general linear model (GLM) to calculate $\mathrm{z}$-score maps using FILM routines (http:// www.fmrib.ox.ac.uk/fsl/).

The following regressors were used to create parameters of images at the individual level: task blocks and contrasts (congruent $>$ neutral, incongruent $>$ neutral and incongruent $>$ congruent). We used the last contrast [incongruent $>$ congruent] in order to probe betweengroup differences, as explained below.

Between-group analysis was conducted using a mixed-effects model (FLAME $1+2$, see references above). Brain activation maps from the two comparisons (MetS $>$ Controls and Controls $>$ MetS) were produced. All images were co-registered to the MNI template. Results were displayed on a surface-based reconstruction produced via a mesh algorithm implemented by MRI3Dx (K. Singh, Cardiff Univ, UK).

In order to analyze BOLD response correlation with cognitive performance on the verbal fluency task after one year we used the MetS group map from the contrast [incongruent $>$ congruent]. A whole brain analysis using an FSL design matrix containing the difference in the $z$-score values of the verbal fluency task was carried out by Pearson's coefficient test corrected for multiple comparisons. We used the FeatQuery algorithm to extract BOLD\% signal change in the areas detected in the thresholded map from the correlation analysis. All FSL maps were considered that passed the threshold of a cluster $\mathrm{z}$ score $>2.3$ and a voxel $\mathrm{p}<0.05$.

\subsection{Statistical analyses}

Data from the neuropsychological assessment were analyzed using the Statistical Package for the Social Sciences 17.0. The non-parametric Mann-Whitney Test was used to compare cognitive performance between groups at baseline and at one-year follow-up. An alpha value of $5 \%$ was used in all statistical analyses.

\section{Results}

The clinical and demographic profile of the population has been previously described in detail (Shigaeff et al., 2013). MetS characteristics were significantly different between the MetS and control groups ( $p<0.01)$. Except for the triglycerides measure, these differences were maintained after one year, despite the presence of drop-outs.

Because hypertension is prevalent in the elderly population, we allocated hypertensive patients equally between the groups to avoid any biases.

\subsection{Neuropsychological data}

The cognitive performance of elderly subjects in both neuropsychological evaluations is shown in Table 1. In the first evaluation (baseline), there was no significant difference in any of the cognitive domains assessed between individuals with and without MetS. However, one year later, MetS subjects showed lower performance in verbal fluency ability compared with controls $(\mathrm{p}=0.02)$. There was no difference between the groups for the other cognitive tests.

\section{2. fMRI data}

During the baseline of the study, three fMRI exams of the volunteers (2MetS and 1 control) presented problems in the analysis and were discarded.

The between-group comparison in the Stroop contrast [incongruent $>$ congruent] showed significant reduction of activity in the MetS group in the left precuneus, right middle occipital gyrus, left inferior temporal gyrus, left anterior cingulated gyrus, both inferior parietal lobules, cerebellum, right superior frontal gyrus, and right medial frontal gyrus (Fig. 1; Table 2). Although not significant, functional activation was also reduced for all other regions compared to controls.

When analyzing verbal fluency after 1 year for the [incongruent $>$ congruent] contrast, and considering all regions, we found a significant negative correlation $\left(\mathrm{R}^{2}=0.13\right)$ between verbal fluency of MetS subjects and the BOLD signal in the right prefrontal cortex (middle frontal gyrus) and the bilateral parietal lobules (border of the intraparietal sulci) after correction for multiple comparisons.

Although we used FDR methods to correct for false positive within the cluster, this approach relies in 5\% false positive voxels. The control for multiple comparisons in fMRI has been subject to constant evaluation. The method we adopted here is prone to higher level of false positive findings then expected. However, authors have noted that the precise estimate if often difficult to obtain, and depend on various types of contrasts and data characteristics (Cox, Chen, Glen, Reynolds, \& Taylor, 2017).

\section{Discussion}

Our results show that the fMRI BOLD response obtained at baseline was reduced for elderly individuals with MetS, even though their neuropsychological performance was similar to that of matched healthy controls. Verbal fluency is associated with the domain of executive function and language, and hence to the activation of fronto-temporoparietal areas (Heinzel et al., 2015; Houvenaghel et al., 2015), the same areas that showed a reduced fMRI response at baseline. We also found a negative correlation between the BOLD signal in the prefrontal and parietal areas at baseline and the cognitive performance of these individuals one year later.

The reduced brain response in the classical Stroop-related activation regions at baseline for cognitively healthy patients with MetS is consistent with previous fMRI findings using a memory task (Hoth et al., 2011) and with our previous findings (Shigaeff et al., 2012), which also showed reduced activation in the prefrontal and parietal areas in these individuals. In interpreting these findings, the role of vascular alterations in the brain of MetS subjects should also be considered. Since fMRI activation is dependent on vascular response, the observed differences in activation may indicate not only (or necessarily) neural differences, but also vascular changes in MetS patients (Portet et al., 2012)

Even though there were no differences in cognitive performance between control and MetS subjects at baseline, for the latter group there was a negative correlation (Fig. 2) between cognitive performance assessed by verbal fluency after one year and the BOLD signal at baseline in the right dorsolateral prefrontal cortex (rDLPFC) and intra-parietal regions (Fig. 3 and Table 3). These findings point to a possible compensatory strategy of the brain: during the baseline test, MetS participants may have engaged these latter regions from the attention network in an attempt to compensate for possible brain tissue burden (as evidenced by the reduced activation observed in the prefrontal and parietal areas) caused by MetS. Such a compensatory mechanism has been suggested for Alzheimer disease (Gleichmann, Chow, \& Mattson, 2011; Kalpouzos, Eustache, \& Desgranges, 2008; Rodda, Dannhauser, Cutinha, Shergill, \& Walker, 2009). We argue that this compensatory mechanism enabled maintenance of the same level of cognitive performance at baseline as was observed in the controls.

Another aspect that can be discussed as a possible explanation for this mechanism is the use of cognitive reserve in the subjects of the MetS group. This phenomenon is characterized by even in the presence of a pathological condition, there is a flexible and efficient solution of the available brain reserve (morphological structure, such as number of neurons for example), in an attempt to maintain cognitive performance satisfactorily (Stern, 2002). There is a wide discussion in the literature evidencing that the experience of the cognitive reserve is directly associated to the level of education of the individual (Bennett et al., 2003; Farfel et al., 2013; Rentz et al., 2010; Sumowski, Chiaravalloti, Krch, Paxton, \& Deluca, 2013). Individuals with higher schooling can cope with a greater neuropathological extent of the disease before the onset 
of symptoms(Stern, Alexander, Prohovnik, \& Mayeux, 1992). However, as already mentioned, a sample of the present study has a low level of schooling, which suggests that their cognitive reserve has a lower limit in comparison with the elderly with higher level of schooling. And because of this limit, this resource can also be depleted more quickly, and then the elderly would finally manifest the cognitive symptoms related to brain damage and present a more rapid cognitive decline (Cheng, 2016). It is known that the incidence of Alzheimer's Dementia is higher in the elderly with low schooling and when the brain can no longer deal with an extension of the pathology, the course of the neurodegenerative pathology can become accelerated (Caramelli et al., 1997; Nitrini et al., 2004; Stern et al., 1994). In this sense, the elderly with MetS would be using the cognitive reserve at the baseline of the study and only a year later they presented the first cognitive symptoms, indicating that there was possibly depleted of this resource.

Nevertheless, regardless of the underlying mechanism, our results suggest that these older adults were already suffering functional brain damage from MetS at baseline, which affected performance over time. Indeed, fMRI responses at baseline were reliable indicators of clinical symptom even in the short term (1 year).

The cognitive decline shown in the follow-up period was insufficient to merit a diagnosis of even mild cognitive impairment (MCI), as these older adults were still functional and did not have relevant complaints of cognitive impairment. However, our results indicate that these individuals may develop MCI over time, and hence should be continuously evaluated to monitor possible disease progression. It is important to emphasize that the clinical relevance of the cognitive symptoms of these neurological diseases may not appear until decades after the onset of brain changes (Lewczuk, Mroczko, Fagan, \& Kornhuber, 2015), highlighting the importance of our findings: the altered activation patterns revealed here can be used as early biomarkers of the onset of cognitive decline, which may ultimately lead to $\mathrm{MCI}$ or dementia.

Our results also encourage more research aimed at defining a possible pattern of brain activation in MetS, and a possible link between MetS and the development of vascular dementia, or even symptoms associated with disorders such as MCI and Alzheimeŕs dementia (de la Torre, 2004).

Some medications are associated with changes in cerebral blood flow (CBF), which may affect the BOLD signal (Restom, Bangen, Bondi, Perthen, \& Liu, 2007). Thus we cannot rule out the possibility that medications taken by MetS subjects altered their brain activation patterns. However, drugs most likely to be associated with changes in BOLD signal are modulators of cardiac activity, and subjects with hypertensive disease were equally distributed between both MetS and control groups. Moreover, because MetS diagnosis requires the presence of three out of five components, each MetS patient had a specific set of prescribed medications, yet changes in BOLD response were consistent for all MetS subjects. This indicates that the altered brain activation patterns observed in this group were likely associated with MetS itself rather than being a by-product of medication use.

Our study is unprecedented in identifying an early biomarker of the possible development of cognitive impairment, particularly in executive function, of elderly individuals suffering from MetS. Our findings also emphasize the importance of satisfactory prevention and remission of metabolic symptoms to prevent the development of cognitive impairment and, ultimately, dementia.

\section{Conflicts of interest}

No other potential conflicts of interest relevant to this article were reported.

\section{Funding}

None.

\section{Acknowledgments}

This research was sponsored by Fundação de Amparo à Pesquisa do Estado de São Paulo (FAPESP) and Coordenação de Aperfeiçoamento de Pessoal de Nivel Superior (CAPES) providing a Masters and $\mathrm{PhD}$ degree fellowship for the first author (Process $\mathrm{N}^{\circ}$ 2009/12271-3). This study also was sponsored by the Ministry of Health which, via the Technical Area of Health of the Aged (PROADI), entered into partnership with the Hospital Israelita Albert Einstein under the Support Program for Institutional Development of the Brazilian Unified National Health System (SUS).

\section{References}

Bennett, D. A., Wilson, R. S., Schneider, J. A., Evans, D. A., Mendes de Leon, C. F., Arnold, S. E., ... Bienias, J. L. (2003). Education modifies the relation of AD pathology to level of cognitive function in older persons. Neurology, 60(12), 1909-1915.

Bruce, D. G., Davis, W. A., Casey, G. P., Starkstein, S. E., Clarnette, R. M., Almeida, O. P., \& Davis, T. M. (2008). Predictors of cognitive decline in older individuals with diabetes. Diabetes Care, 31(11), 2103-2107. http://dx.doi.org/10.2337/dc08-0562.

Brucki, S., Nitrini, R., Caramelli, P., Bertolucci, P. H., \& Okamoto, I. H. (2003). Sugestões para o uso do mini-exame do estado mental no Brasil. Arquivos de Neuro-Psiquiatria, 61(3B).

Caramelli, P., Poissant, A., Gauthier, S., Bellavance, A., Gauvreau, D., Lecours, A. R., \& Joanette, Y. (1997). Educational level and neuropsychological heterogeneity in dementia of the Alzheimer type. Alzheimer Disease and Associated Disorders, 11(1), 9-15.

Cavalieri, M., Ropele, S., Petrovic, K., Pluta-Fuerst, A., Homayoon, N., Enzinger, C., ... Schmidt, R. (2010). Metabolic syndrome, brain magnetic resonance imaging, and cognition. Diabetes Care, 33(12), 2489-2495. http://dx.doi.org/10.2337/dc10-0851.

Cheng, S. T. (2016). Cognitive reserve and the prevention of dementia: The role of physical and cognitive activities. Current Psychiatry Reports, 18(9), 85. http://dx.doi. org/10.1007/s11920-016-0721-2.

Collinson, S. L., Tong, S. J., Loh, S. S., Chionh, S. B., \& Merchant, R. A. (2014). Midlife metabolic syndrome and neurocognitive function in a mixed Asian sample. International Psychogeriatrics, 26(8), 1305-1316. http://dx.doi.org/10.1017/ S104161021400057X.

Cox, R. W., Chen, G., Glen, D. R., Reynolds, R. C., \& Taylor, P. A. (2017). FMRI clustering in AFNI: False-positive rates redux. Brain Connectivity, 7(3), 152-171. http://dx.doi. org/10.1089/brain.2016.0475.

Creavin, S. T., Gallacher, J., Bayer, A., Fish, M., Ebrahim, S., \& Ben-Shlomo, Y. (2012). Metabolic syndrome, diabetes, poor cognition, and dementia in the Caerphilly prospective study. Journal of Alzheimer Disease, 28(4), 931-939. http://dx.doi.org/10. 3233/JAD-2011-111550.

de la Torre, J. C. (2004). Is Alzheimer's disease a neurodegenerative or a vascular disorder? Data, dogma, and dialectics. Lancet Neurology, 3(3), 184-190. http://dx.doi. org/10.1016/S1474-4422(04)00683-0.

De Silva, D. A., Woon, F. P., Lee, M. P., Chen, C. L., Chang, H. M., \& Wong, M. C. (2009). Metabolic syndrome is associated with intracranial large artery disease among ethnic Chinese patients with stroke. Journal of Stroke and Cerebrovascular Diseases, 18(6), 424-427. http://dx.doi.org/10.1016/j.jstrokecerebrovasdis.2009.01.010.

Dik, M. G., Jonker, C., Comijs, H. C., Deeg, D. J., Kok, A., Yaffe, K., \& Penninx, B. W. (2007). Contribution of metabolic syndrome components to cognition in older individuals. Diabetes Care, 30(10), 2655-2660. http://dx.doi.org/10.2337/dc06-1190.

Farfel, J. M., Nitrini, R., Suemoto, C. K., Grinberg, L. T., Ferretti, R. E., Leite, R. E., ... B.A.B.S. Group (2013). Very low levels of education and cognitive reserve: A clinicopathologic study. Neurology, 81(7), 650-657. http://dx.doi.org/10.1212/WNL. 0b013e3182a08f1b.

Ford, E. S., Giles, W. H., \& Dietz, W. H. (2002). Prevalence of the metabolic syndrome among US adults: Findings from the third National Health and Nutrition Examination Survey. JAMA, 287(3), 356-359.

Gleichmann, M., Chow, V. W., \& Mattson, M. P. (2011). Homeostatic disinhibition in the aging brain and Alzheimer's disease. Journal of Alzheimer's Disease, 24(1), 15-24. http://dx.doi.org/10.3233/JAD-2010-101674.

Golden, C., \& Freshwater, S. (2002). Stroop color and word test: Revised examiner's manual. In W. Dales (Ed.), Stoelting.

Grundy, S. M., Cleeman, J. I., Daniels, S. R., Donato, K. A., Eckel, R. H., Franklin, B. A., ... National Heart, L. n., and Blood Institute (2005). Diagnosis and management of the metabolic syndrome: An American Heart Association/National Heart, Lung, and Blood Institute Scientific Statement. Circulation, 112(17), 2735-2752. http://dx.doi. org/10.1161/CIRCULATIONAHA.105.169404.

Haley, A. P., Gonzales, M. M., Tarumi, T., \& Tanaka, H. (2012). Subclinical vascular disease and cerebral glutamate elevation in metabolic syndrome. Metabolic Brain Disease, 27(4), 513-520. http://dx.doi.org/10.1007/s11011-012-9306-x.

Heinzel, S., Metzger, F. G., Ehlis, A. C., Korell, R., Alboji, A., Haeussinger, F. B., ... Fallgatter, A. J. (2015). Age and vascular burden determinants of cortical hemodynamics underlying verbal fluency. PLoS One, 10(9), e0138863. http://dx.doi.org/10. 1371/journal.pone.0138863.

Hoth, K. F., Gonzales, M. M., Tarumi, T., Miles, S. C., Tanaka, H., \& Haley, A. P. (2011). Functional MR imaging evidence of altered functional activation in metabolic syndrome. AJNR American Journal of Neuroradiology, 32(3), 541-547. http://dx.doi.org/ 10.3174/ajnr.A2315. 
Houvenaghel, J. F., Le Jeune, F., Dondaine, T., Esquevin, A., Robert, G. H., Péron, J., ... Sauleau, P. (2015). Reduced verbal fluency following subthalamic deep brain stimulation: A frontal-related cognitive deficit? PLoS One, 10(10), e0140083. http://dx. doi.org/10.1371/journal.pone.0140083.

Kalpouzos, G., Eustache, F., \& Desgranges, B. (2008). Cognitive reserve and neural networks in normal aging and Alzheimer's disease. Psychologie and Neuropsychiatrie du Vieillissement, 6(2), 97-105. http://dx.doi.org/10.1684/pnv.2008.0120.

Kozasa, E. H., Sato, J. R., Lacerda, S. S., Barreiros, M. A., Radvany, J., Russell, T. A., ... Amaro, E. (2012). Meditation training increases brain efficiency in an attention task. Neuroimage, 59(1), 745-749. http://dx.doi.org/10.1016/j.neuroimage.2011.06.088.

Lewczuk, P., Mroczko, B., Fagan, A., \& Kornhuber, J. (2015). Biomarkers of Alzheimer's disease and mild cognitive impairment: A current perspective. Advances in Medical Sciences, 60(1), 76-82. http://dx.doi.org/10.1016/j.advms.2014.11.002.

Luchsinger, J. A., Tang, M. X., Stern, Y., Shea, S., \& Mayeux, R. (2001). Diabetes mellitus and risk of Alzheimer's disease and dementia with stroke in a multiethnic cohort. American Journal of Epidemiology, 154(7), 635-641.

McEvoy, L. K., Laughlin, G. A., Barrett-Connor, E., Bergstrom, J., Kritz-Silverstein, D., Der-Martirosian, C., \& von Mühlen, D. (2012). Metabolic syndrome and 16-year cognitive decline in community-dwelling older adults. Annals of Epidemiology, 22(5), 310-317. http://dx.doi.org/10.1016/j.annepidem.2011.12.003.

McGuire, L. C., Ford, E. S., \& Ajani, U. A. (2006). The impact of cognitive functioning on mortality and the development of functional disability in older adults with diabetes: The second longitudinal study on aging. BMC Geriatrics, 6, 8. http://dx.doi.org/10. 1186/1471-2318-6-8.

Nitrini, R., Caramelli, P., Herrera, E., Bahia, V. S., Caixeta, L. F., Radanovic, M., .. Takahashi, D. Y. (2004). Incidence of dementia in a community-dwelling Brazilian population. Alzheimer Disease and Associated Disorders, 18(4), 241-246.

Niwa, H., Koumoto, C., Shiga, T., Takeuchi, J., Mishima, S., Segawa, T., ... Yoshioka, N. (2006). Clinical analysis of cognitive function in diabetic patients by MME and SPECT. Diabetes Research and Clinical Practice, 72(2), 142-147. http://dx.doi.org/10. 1016/j.diabres.2005.10.012.

Park, J. H., \& Kwon, H. M. (2008). Association between metabolic syndrome and previous ischemic lesions in patients with intracranial atherosclerotic stroke. Clinical Neurology and Neurosurgery, 110(3), 215-221. http://dx.doi.org/10.1016/j.clineuro.2007.10. 016.

Portet, F., Brickman, A. M., Stern, Y., Scarmeas, N., Muraskin, J., Provenzano, F. A., ... Akbaraly, T. N. (2012). Metabolic syndrome and localization of white matter hyperintensities in the elderly population. Alzheimer's \& Dementia, 8(Suppl. 5), S88-S95. http://dx.doi.org/10.1016/j.jalz.2011.11.007 e81.

Raffaitin, C., Féart, C., Le Goff, M., Amieva, H., Helmer, C., Akbaraly, T. N., ... BarbergerGateau, P. (2011). Metabolic syndrome and cognitive decline in French elders: The Three-City Study. Neurology, 76(6), 518-525. http://dx.doi.org/10.1212/WNL. Ob013e31820b7656.

Rentz, D. M., Locascio, J. J., Becker, J. A., Moran, E. K., Eng, E., Buckner, R. L., ... Johnson, K. A. (2010). Cognition, reserve, and amyloid deposition in normal aging.
Annals of Neurology, 67(3), 353-364. http://dx.doi.org/10.1002/ana.21904.

Restom, K., Bangen, K. J., Bondi, M. W., Perthen, J. E., \& Liu, T. T. (2007). Cerebral blood flow and BOLD responses to a memory encoding task: A comparison between healthy young and elderly adults. Neuroimage, 37(2), 430-439. http://dx.doi.org/10.1016/j. neuroimage.2007.05.024.

Rigo, J. C., Vieira, J. L., Dalacorte, R. R., \& Reichert, C. L. (2009). Prevalence of metabolic syndrome in an elderly community: Comparison between three diagnostic methods. Arquivos Brasileiros de Cardiologia, 93(2), 85-91.

Roberts, R. O., Geda, Y. E., Knopman, D. S., Cha, R. H., Boeve, B. F., Ivnik, R. J., ... Petersen, R. C. (2010). Metabolic syndrome, inflammation, and nonamnestic mild cognitive impairment in older persons: A population-based study. Alzheimer Disease and Associated Disorders, 24(1), 11-18. http://dx.doi.org/10.1097/WAD. 0b013e3181a4485c.

Rodda, J. E., Dannhauser, T. M., Cutinha, D. J., Shergill, S. S., \& Walker, Z. (2009). Subjective cognitive impairment: Increased prefrontal cortex activation compared to controls during an encoding task. International Journal of Geriatric Psychiatry, 24(8), 865-874. http://dx.doi.org/10.1002/gps.2207.

Rouch, I., Trombert, B., Kossowsky, M. P., Laurent, B., Celle, S., Ntougou Assoumou, G., ... Barthelemy, J. C. (2014). Metabolic syndrome is associated with poor memory and executive performance in elderly community residents: The PROOF study. American Journal of Geriatric Psychiatry, 22(11), 1096-1104. http://dx.doi.org/10.1016/j.jagp. 2014.01.005.

Shigaeff, N., Jacinto, A., Chiochetta, G., Cendoroglo, M. S., Amaro, E., Ikeda, M., ... Citero, V. (2012). Cognition and cerebral neurovascular coupling in the elderly with metabolic syndrome. Alzheimer's and Dementia: The Journal of the Alzheimer's Association Supplement, 8(4).

Shigaeff, N., Jacinto, A. F., Franco, F. G., Chiochetta, G., Cendoroglo, M. S., \& Citero, V. A. (2013). Cognitive assessment in an elderly population with metabolic syndrome in Brazil. Dementia \& Neuropsychologia, 7(2).

Stern, Y., Alexander, G. E., Prohovnik, I., \& Mayeux, R. (1992). Inverse relationship between education and parietotemporal perfusion deficit in Alzheimer's disease. Annals of Neurology, 32(3), 371-375. http://dx.doi.org/10.1002/ana.410320311.

Stern, Y., Gurland, B., Tatemichi, T. K., Tang, M. X., Wilder, D., \& Mayeux, R. (1994) Influence of education and occupation on the incidence of Alzheimer's disease. JAMA, 271(13), 1004-1010.

Stern, Y. (2002). What is cognitive reserve? Theory and research application of the reserve concept. Journal of the International Neuropsychological Society, 8(3), 448-460.

Sumowski, J. F., Chiaravalloti, N., Krch, D., Paxton, J., \& Deluca, J. (2013). Education attenuates the negative impact of traumatic brain injury on cognitive status. Archives of Physical Medicine and Rehabilitation, 94(12), 2562-2564. http://dx.doi.org/10. 1016/j.apmr.2013.07.023.

Zhang, C. E., van Raak, E. P., Rouhl, R. P., Lodder, J., Staals, J., Knottnerus, I. L., \& van Oostenbrugge, R. J. (2010). Metabolic syndrome relates to lacunar stroke without white matter lesions: A study in first-ever lacunar stroke patients. Cerebrovascular Diseases, 29(5), 503-507. http://dx.doi.org/10.1159/000297967. 